\title{
Seminoma primário do mediastino
}

\author{
Primary mediastinal seminoma
}

\author{
Cleverson Winston de Liz Medeiros', Cesar Giovani Conte'2, Gilberto Tokunaga², Ivo Batista Júnior', \\ Lúcia Noronha ${ }^{3}$ e Calixto Antônio Hakim Neto ${ }^{4}$
}

\section{Resumo}

O seminoma primário de mediastino é uma neoplasia rara, principalmente em mulheres, nas quais existem apenas oito casos descritos de seminoma. Tem sua maior incidência entre a terceira e quarta décadas, caracterizando-se por grande radiossensibilidade, sendo a quimioterapia com drogas como cisplatina ou carboplatina utilizada em casos de doença avançada.

O s autores relatam um caso de seminoma de mediastino em paciente feminina de 76 anos de idade que apresentava como sintomas iniciais dor torácica, tosse produtiva e dispnéia aos médios esforços. 0 diagnóstico foi firmado por imagem e toracotomia, além da alteração dos marcadores séricos característicos de tumores de células germinativas. D evido à doença estar localmente avançada, o tratamento proposto foi quimioterapia à base de cisplatina, mas a paciente evoluiu com insuficiência respiratória grave e óbito.

Os autores fazem uma revisão da incidência, patogênese, diagnóstico e formas atuais de tratamento do seminoma de mediastino, além da classificação prognóstica proposta pelo International Germ Cell Cancer Collaborative Group.

Palavras-chave: seminoma; neoplasias do mediastino; quimioterapia; diagnóstico.

\footnotetext{
${ }^{1}$ M édico Residentedo Serviço deO ncologia do H ospital N ossa Senhora das G raças. Enviar correspondência para C.W.L.M . Praça G eneral O sório 205, apto 404; 80020-010 Curitiba, PR - Brasil.E-mail: cleversonwinston@yahoo.com

${ }^{2}$ A cadêmico Interno do H ospital N ossa Senhora das G raças e da U niversidade Federal do Paraná

${ }^{3} \mathrm{M}$ édica Patologista do H ospital N ossa Senhora das G raças.

${ }^{4} \mathrm{M}$ édico $\mathrm{C}$ hefe do Serviço de O ncologia do H ospital N ossa Senhora das G raças.

Recebido em abril de 2001
} 


\begin{abstract}
The primary mediastinal seminoma is a rare disease, mainly in women. There are only eight cases of seminoma described in the English literature. The disease has a greater incidence in the third and fourth decades of life. An important characteristic is the radio sensibility of the tumor, while cisplatin or carboplatin based chemotherapy is applied for advanced disease.

The authors describe a 76-year-old female patient with mediastinal seminoma. $\mathrm{H}$ er first symptoms were chest pain, cough with sputum and dyspnea in median efforts. I mage exams and thoracotomy performed her diagnostic, added to characteristic alterationsin the germ cell tumor-markers. Cisplatin was the proposed treatment, but she had a severe respiratory failure and died.

The authors review the incidence, pathogenesis, diagnosis, treatments to the mediastinal seminoma and a prognostic classification proposed by International Germ Cell Cancer Collaborative Group.
\end{abstract}

Key words: seminoma; mediastinal neoplasms; chemotherapy; diagnostic.

\section{INTRODUÇÃO}

D entreostumores de células germinativas, os de mediastino são raros, correspondendo entre $1 \%$ e $3 \% .^{1,2}$ Estes tumores têm maior incidência entre a terceira e quarta décadas, sendo extremamente raros em mulheres, com 33 casos relatados de tumores nãoseminomatosos ${ }^{3}$ e apenas oito casos de tumores seminomatosos. ${ }^{4-9}$

O s sintomas mais freqüentes são dor torácica, dispnéia e tosse. O s tumores seminomatosos são usualmente grandes e freqüentemente irressecáveis, mas têm como característica boa resposta ao tratamento radioterápico. Atualmente, o tratamento quimioterápico com cisplatina ou carboplatina ${ }^{10-12}$ também tem sido utilizado em pacientes com doença avançada.

O s autores relatam um caso de seminoma primário de mediastino em paciente feminina de 76 anos.

\section{RELATO DE CASO}

Paciente feminina, 76 anos, branca, procurou assistência médica queixando-se de tosse produtiva, associada a dor torácica ventilatório-dependentee dispnéia aos médios esforços, com evolução de cinco meses. Era previamente hígida e apesar da idade, não apresentava outras co-morbidades. $\mathrm{N}$ a história familiar, tinha o pai falecido por câncer de estômago. A paciente foi tabagista de 20 cigarros/dia por 40 anos.

Estava em bom estado geral e ao exame físico segmentar apresentava redução do murmúrio vesicular em base de hemitórax esquerdo com sibilos difusos e estertores bolhosos à direita, sem outras alterações relevantes. A radiografia e a tomografia de tórax demonstraram lesão em terço inferior de pulmão esquerdo com massa peri-hilar de limites imprecisos paracardíaco esquerdo e adenomegalias paracarinais. A tomografia de abdômen estava dentro dos padrões normais. Pela broncoscopia observou-se compressão extrínseca de brônquio do lobo inferior esquerdo e lesão vegetante obstrutiva de brônquio da língula. 0 diagnóstico histopatológico desta lesão e do lavado broncoalveolar foram negativos para malignidade. A punção aspirativa com agulha fina (PAAF) guiada por tomografia foi inconclusiva.

Pela presença de massa pulmonar com compressão de brônquio de lobo inferior esquerdo e sem diagnóstico, ela foi submetida à toracotomia póstero-lateral esquerda. $\mathrm{Na}$ 


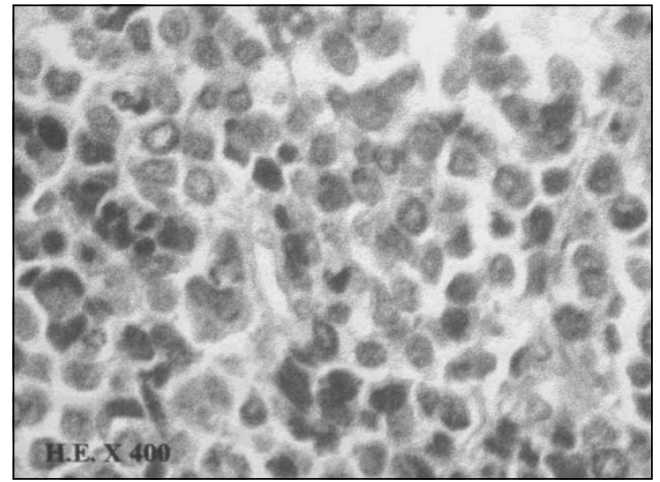

Figura 1. Microfotografia de seminoma mediastinal ( $\mathrm{HE}$ $400 x)$

cirurgia foi observado derrame pleural volumoso, congestão e compressão de lobo inferior esquerdo, além de tumoração friável envolvendo hilo pulmonar esquerdo. 0 exame por congelação desta lesão revelou neoplasia pouco diferenciada. Pela indeterminação do diagnóstico e pelas características macroscópicas que lembrava linfoma, optouse por aguardar o exame anátomo-patológico definitivo. Ela permaneceu sob ventilação mecânica no pós-operatório durante sete dias, devido à dificuldade respiratória, evoluindo com derrame pleural extenso à esquerda e condensações de aspecto inflamatório no pulmão esquerdo.

0 exame histopatológico (Figura 1 ) revelou neoplasia pouco diferenciada, sendo a seguir realizada imuno-histoquímica (Tabela 1) que favoreceu o diagnóstico de seminoma.

O s marcadores séricos apresentaram desidrogenase lática (LD H) elevada, mas com alfafetoproteína (AFP) e gonadotrofina coriônica humana (H CG) normais (Tabela 2).

D evido ao grande volume do tumor, com invasão de hilo e parênquima pulmonar, além de comprometimento delinfonodos regionais, optou-se por quimioterapia.

Tabela 1. Resultado da imuno-histoquímica

\begin{tabular}{|c|c|}
\hline Nactotor & Resalods \\
\hline 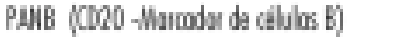 & Negotion \\
\hline EMM (Untigero de nentrons apitelio) & $+/-$ \\
\hline 630 & Neguting \\
\hline EME (andase neuvinis expeofica) & ++ \\
\hline ICA (antigan kuxtóttin ctenum) & Neguin \\
\hline PAIT (CD45 R0 - mocedor de caldes T) & Negotim \\
\hline 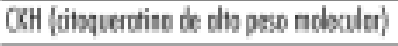 & Negtim \\
\hline VIV (̧̧inetrine) & hondasive \\
\hline
\end{tabular}

Ela reinternou após três semanas com queda do estado geral, dispnéia aos esforços, astenia e sinais de derrame pleural extenso à esquerda. Foi realizado toracocentese de alívio com drenagem de líquido amarelo citrino, de moderada quantidade, com melhora do quadro de dispnéia, porém a paciente foi mantida sob oxigenoterapia contínua. Ela evoluiu com episódios recorrentes de broncoespasmo e finalmente com insuficiência respiratória aguda, sendo transferida para UTI, onde ocorreu parada cardiorrespiratória e óbito.

\section{DISCUSSÃO}

O s tumores de células germinativas podem ser benignos (teratomas) ou malignos. O s tumores malignos são classificados em seminoma e não-seminoma (carcinoma embrionário, teratoma imaturo, tumor de saco vitelino, coriocarcinoma e tumores mistos). O s tumores primários extragonadais são raros, e podem se originar virtualmente em qualquer sítio, mas preferencialmente em estruturas da linha média como mediastino, retroperitônio, glândula pineal e região sacrococcígea. ${ }^{13}$

0 mediastino anterior é o sítio primário extragonadal mais freqüentemente acometido pelos tumores de células germinativas, representando $50 \%$ a $70 \%$ de todos os tumores extragonadais. ${ }^{2,14} 0$ seminoma é 0 tipo histológico maligno mais comum, correspondendo a $37 \%,{ }^{15}$ Em estudo realizado por M oran et al. ${ }^{8} \mathrm{em} 120$ pacientes com seminoma de mediastino, a maioria dos pacientes $(70,8 \%)$ desenvolve a doença entre a terceira e quarta década de vida, todos eram homens e apenas um tinha idade superior a 70 anos. Em mulheres há apenas oito casos descritos de seminoma primário de mediastino. 1,4-7,9,16

\begin{tabular}{|c|c|}
\hline Narador & Velar sétices \\
\hline LDH & $\begin{array}{c}710 \text { แ/ (valores nunais de atréncio: } 89 \text { o } 240 \\
\text { U/) }\end{array}$ \\
\hline AFP & 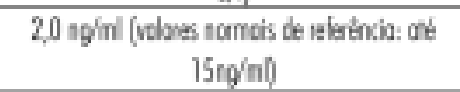 \\
\hline$H=6$ & 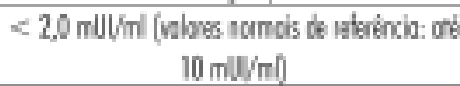 \\
\hline
\end{tabular}

Clinicamente, o seminoma se apresenta como massa de mediastino anterior. Os 
principais sintomas são dor torácica subesternal, dispnéia e tosse, estando diretamente relacionado à compressão de estruturas adjacentes pelo tumor. A síndrome de veia cava superior pode se manifestar tardiamente em $9 \%$ a $26 \%$ dos pacientes. ${ }^{1,8}$ 0 casionalmente os pacientes apresentam como primeira manifestação a doença metastática. 0 sítio metastático mais freqüente é o tórax (pulmões e linfonodos regionais), seguido de ossos. 0 utros possíveis sítios de metástase são fígado, baço, amídala, tireóide, adrenal, pele e sistema nervoso central.6,9 O seminoma de mediastino pode ser totalmente assintomático em $20 \%$ a $30 \%$ dos casos, sendo encontrado incidentalmente em estudos radiográficos de rotina. ${ }^{8,9}$

Os marcadores tumorais séricos (HCG, AFP eLD H) são indispensáveis para o diagnóstico dos tumores de células germinativas. 0 seminoma primário de mediastino pode estar associado à baixa elevação do HCG (usualmente abaixo de $100 \mathrm{mUl} / \mathrm{ml}$ ) e seu diagnóstico requer níveis normais de AFP. Elevações significativas de AFP pode indicar a presença de componente tumoral não-seminomatoso. ${ }^{2}$ O LDH está elevado nos tumores seminomas e não seminomas.

$\mathrm{H}$ istologicamente, os seminomas de mediastino são semelhantes aos seminomas gonadais. Reação granulomatosa, células gigantes multinucleares esparsas, e células trofoblásticas gigantes ocasionais são comuns a ambos. Porém o seminoma de mediastino pode apresentar algumas características histológicas próprias, como a presença de restos de tecido tímico em áreas centrais ou periféricas do tumor e alterações císticas evidentes simulando cisto multilocular de timo. ${ }^{17}$

0 diagnóstico diferencial inclui uma variedade de neoplasias (primárias e metastáticas) de mediastino, portanto além de história clínica detalhada, o estudo imunohistoquímico pode fornecer dados importantes para o diagnóstico. $N$ ão é infreqüente que 0 diagnóstico só se estabeleça com toracotomia, como relataram Bush et al. ${ }^{18}$ e Cox et al. ${ }^{4} \mathrm{em}$ que 10 de 13 pacientes e 19 de 23 pacientes respectivamente obtiveram diagnóstico após esse procedimento. M etástase de seminoma primário das gônadas deve ser sempre considerado, porém poucos casos foram documentados, sendo extremamenteraro. M ela- noma maligno metastático também deve ser considerado, principalmente quando a amostra biopsiada é pouco representativa. Em relação aos tumores primários de mediastino, os mais freqüentemente envolvidos são o timoma, carcinoma de timo, linfoma de $\mathrm{H}$ odgkin e outros tumores de células germinativas.

M uitas teorias foram propostas para explicar a patogênese do seminoma de mediastino. Três delas defendem a origem extragonadal do tumor, enquanto uma quarta defende que o seminoma de mediastino representa uma metástase de tumores testiculares ocultos. A primeira hipótese, proposta por Schlumberger, ${ }^{19}$ sugere que 0 tumor tem origem intratímica resultante de uma desordem do desenvolvimento somático na embriogênese. A íntima associação do ectomesoderma com elementos do endoderma durante a timogênese, seria responsável pela presença dos três folhetos embrionários nos teratomas de mediastino. Porém, esta hipótese não explica a presença de elementos germinativos nos tumores mistos. ${ }^{9}$ Friedman et al. ${ }^{20}$ propõe que todos os tumores extragonadais tem origem nas células germinativas primordiais amplamente distribuídas nos diversos sítios durante a embriogênese (timo, cérebro, fígado, medula óssea), carreando informações genéticas ou exercendo funções somáticas reguladoras nestes locais. ${ }^{21}$ Portanto os diferentes tipos histológicos resultariam da tumorigênese destas células nos diferentes estágios de diferenciação. Estudos embriológicos sustentam esta hipótese, demonstrando ser possível a presença de células germinativas primordiais no retroperitôneo e mediastino. Por outro lado, baseados em estudos comparativos de alterações citogenéticas de tumores primários mediastinais e gonadais, C haganti et al. ${ }^{22}$ sugeriram que estes tumores teriam origem da migração de células germinativas gonadais transformadas, e não de células germinativas primordiais distribuídas durante a embriogênese como proposto por Friedman. ${ }^{21}$ Uma quarta hipótese defende a possível metástase de tumores testiculares ocultos, entretanto, metástases para o mediastino anterior de tumores testiculares são extremamente raras, sendo que elas normalmente ocorrem inicialmente para retroperitôneo e apenas 
posteriormente para o mediastino; outra crítica a essa teoria é que esses tumores também ocorrem em pacientes femininas, ${ }^{2,4,10}$ como no presente caso.

0 tratamento ainda permanece em discussão. 0 seminoma de mediastino se assemelha com o seminoma gonadal tanto pelas características histológicas quanto por sua radiossensibilidade. Aygun et al., ${ }^{23}$ propõe ressecção tumoral primária isolada, seguido de radioterapia locorregional somente para os pacientes cuja cirurgia foi limitada à biópsia ou ressecção incompleta, reservando a quimioterapia aos pacientes com metástase à distância comprovada. Lemarié et al. ${ }^{1}$ recomendam a ressecção cirúrgica e posterior radioterapia, e defendem que só existe benefício real com a cirurgia se a excisão for completa. $\mathrm{N}$ ichols et al., ${ }^{2}$ por outro lado, recomendam que os pacientes com seminoma isolado de mediastino sejam tratados com radioterapia somente. Porém, devido à sua localização no mediastino anterior, que permite crescimento tumoral maior com poucos sintomas, estes tumores podem se apresentar já com invasão de estruturas intratorácicas adjacentes e com extensão para o hilo e parênquima pulmonar. N estes casos pode ser difícil o tratamento radioterápico ou resultar em irradiação excessiva nas estruturas mediastinais e no parênquima pulmonar normal. Portanto os pacientes com tumor avançado ou com doença metastática extra-mediastinal, como no presente caso, teriam maior benefício com quimioterapia baseada em cisplatina ${ }^{1,2}$ ou carboplatina. ${ }^{10-12}$

Freqüentemente se observam massas residuais pós-quimioterapia, principalmente em seminomas avançados, mas seu manejo permanece controverso. Em estudo realizado por Puc et al., ${ }^{24}$ com 104 pacientes com seminoma avançado que apresentavam massa re sidual pós-quimioterapia, $90 \%$ das lesões se constituíam de fibrose e necrose, não havendo recidiva local. Porém massas residuais maiores quetrês centímetros $(\mathrm{cm})$ apresentavam células tumorais em $27 \%$ dos casos, ao contrário dos $3 \%$ presentes nas lesões menores que três $\mathrm{cm}$. Portanto, propõe-se a excisão cirúrgica sempre quando possível, nas lesões residuais maiores que $3 \mathrm{~cm}$.

O seminoma de mediastino apresenta melhor prognóstico que tumores mediastinais não-seminoma, e pior em relação aos seminomas gonadais, ${ }^{25}$ pois: no testículo, a túnica albugínea envolve 0 tumor e evita disseminação precoce; no mediastino, o tumor geralmente se apresenta em estadio mais avançado, por apresentar poucos sintomas, mesmo quando relativamentegrandes; ehaver a tendência à disseminação hematogênica, tendo em vista sua localização.

G enericamente, os pacientes portadores de seminoma de mediastino têm sobrevida em 5 anos de $75 \% .^{6,9}$ os pacientes que parecem ter um pior prognóstico são aqueles que no momento do diagnóstico têm 37 anos ou mais, ${ }^{8}$ síndrome da veia cava superior, envolvimento linfonodal cervical ou supraclavicular, evidência radiológica de envolvimento hilar, ou febre como sintoma inicial. ${ }^{6}$

Em 1997 foi proposta uma classificação prognóstica para os tumores de células germinativas pelo International Germ Cell Cancer Collaborative G roup. ${ }^{26} \mathrm{O}$ s tumores são classificados em três grupos: bom prognóstico, prognóstico intermediário e mau prognóstico; tomando-se como fatores o sítio primário, a presença de metástase visceral não pulmonar e os níveis de marcadores séricos tumorais (AFP, HCG, LDH). Em relação ao seminoma, $90 \%$ são classificados como sendo de bom prognóstico, independente do sítio primário, desde que não possuam metástase visceral, exceto pulmonar - sobrevida de $86 \%$ em cinco anos. Outros 10\% correspondem aos tumores com prognóstico intermediário, com metástase visceral não pulmonar sobrevida de $72 \%$ em 5 anos. 0 seminoma não se classifica como tendo mau prognóstico.

O seminoma primário de mediastino é um tumor raro, especialmente em mulheres, geralmente de bom prognóstico. 0 caso aqui relatado evoluiu mal devido à insuficiência respiratória aguda, o que privou a paciente do tratamento quimioterápico, levando-a ao óbito.

\section{REFERÊNCIAS BIBLIOGRÁFICAS}

1. LemariéE, Assouline PS, Diot $P$, Regnard JF, Levasseur $P, D$ roz JP, RuffieP. Primary mediastinal germ cell tumors: results of a French retrospective study. Chest 1992;102(5):1477-83. 
2. N icholsCR. M ediastinal germ cell tumors: clinical features and biologic correlates. Chest 1991;99:472-9.

3. M orishimaY, Satoh H, O htsukaM, YazawaT, YamashitaY, H asegawaS. Primary mediastianal nonseminomatous germ cell tumour in an adult female. Resp M ed 1998; 92(6):882-4.

4. CoxJD. Primary malignant germinal tumors of the mediastinum: astudy of 24 cases. C ancer 1975;36:1162-8.

5. El D omeiri AA, H utter RV, Pool JL, FooteFW. Primary mediastinal seminoma of the anterior mediastinum. Ann T horac Surg 1968;6:513-21.

6. H urt RD, Bruckman JE, Farrow GM , Bernatz $P E, H$ ahn RG, EargleJD. Primary anterior mediastinal seminoma. C ancer 1982;49:1658-63.

7. I verson L. Thymoma: a review a reclassification. Am J Pathol 1956;32:695.

8. M oran CA, Suster S, Przygodzki RM, Koss $M N$. Primary germ cell tumors of themediastinum. II. M ediastinal seminomas: aclinicopathologic and immunohistochemical study of 120 cases. C ancer 1997;80:691-8.

9. Polanski SM, Barwick KW, Ravin CE. Primary mediastinal seminoma. AJR 1979; 132:17-21.

10. ChildsWJ, Goldstraw P, N icholsJE, D ernaley D P, H orwich A. Primary malignant mediastinal germ cell tumors: improved prognosiswith platinum-based chemotherapy and surgery. $\mathrm{Br}$ J Cancer 1993;67:1098-101.

11. H owich A, D earnaley DP, D uchesne GM , W illiams M, Brada M , Peckham M J. Simple nontoxic treatment of advanced metastatic seminoma with carboplatin. J Clin O ncol 1989;7:1150-6.

12. Peckham MJ, H orwich A, Brada M, Drury A, $H$ endry W F. Cis-diamine-1,1-cyclobutane dicarboxylateplatinum II (carboplatin) in thetreatment of testicular germ-cell tumours: apreliminary report. CancerTreat Rev 1985;12:101-10.

13. Jacob R, Ramadas K, Jyothirmayi R, Kusumakumary P, Nair M K. Extragonadal germ cell tumors: aten years experience. Am J Clin Oncol 1998;21(2):198-202.
14. LajosT Z, CharretteEJ , Farr JA. Primary mediastinal seminoma. Chest 1971;59:575-8.

15. Schantz A, Sewall W, C astlemen B. M ediastinal germinoma: a study of 21 cases with an excellent prognosis. C ancer 1972;30:1189-94.

16. M artini N , G olbey RB, H adju SI, W hitmore W F, BeattieEJ Jr. Primary mediastinal germ cell tumors. C ancer 1974;33:763-9.

17. M oran CA, Suster S. M ediastinal seminomas with prominent cystic changes: a clinicopathologic study of 10 cases. Am J Surg Pathol 1995;19(9):1047-53.

18. Bush SE, M artinezA, Babshaw M A. Primaryme diastinal seminoma. Cancer 1981; 48:1877-82.

19. Schlumberger H G. Teratoma of the anterior mediastinum in the group of military age: a study of sixteen cases, and a review of theories of genesis. Arch Pathol 1946; 41:398-444.

20. Friedman N B. The comparativemorphogenesis of extragenital and gonadal teratoid tumors. C ancer 1951;4:265-76.

21. Friedman $\mathrm{N}$. The function of the primordial germ cell in extragonadal tissues. Int J Androl 1987;10:43-9.

22. Chaganti RS, H ouldsworthJ. The cytogenetic theory of thepathogenesis of human adult male germ cell tumors: review article. APMIS 1998;106(1):80-4.

23. Aygun C, Slawson RG , Bajaj K, Salazar OM . Primary mediastinal seminoma. U rology 1984;23:109-17.

24. Puc H S, H eelan R, M azumdar $M, H$ err $H$, Scheinfeld J, Vlamis V, Bajorin DF, Bosl GJ, $M$ encel $P, M$ otzer $R J$. $M$ anagement of residual mass in advanced seminoma: results and recommendations from the Memorial SloanKettering $\mathrm{C}$ ancer $\mathrm{C}$ enter. J Clin $\mathrm{O}$ ncol 1996;14(2):454-60.

25. Raghavan $D$, Barrett $A$. M ediastinal seminomas. Cancer 1980;46:1187-91.

26. Internatioanl Germ C ell Cancer Collaborative Group. International G erm C ell Consensus Classification: a prognostic factor-based staging system for metastic germ cell cancers. J C lin O ncol 1997;15(2):594-603. 